\section{ORIGINAL RESEARCH}

\author{
S.-T. Park \\ J.K. Kim \\ K.H. Yoon \\ S.-O. Park \\ S.W. Park \\ J.S. Kim \\ S.J. Kim \\ D.C. Suh
}

\title{
Atherosclerotic Carotid Stenoses of Apical versus Body Lesions in High-Risk Carotid Stenting Patients
}

BACKGROUND AND PURPOSE: Different lesion locations in the atherosclerotic carotid bulb stenosis have not been clearly defined. We sought to evaluate 2 locations of carotid bulb stenosis in high-risk patients and to determine the relationship of each location to atherosclerotic risk factors and clinical features.

MATERIALS AND METHODS: Atherosclerotic carotid plaques of apical versus body lesions, defined according to the area and extent of plaque involvement, were retrospectively analyzed in 200 consecutive high-risk patients who underwent carotid stent placement because of $\geq 50 \%$ symptomatic stenosis. We evaluated interobserver concordance and assessed each type of lesion relative to 13 atherosclerotic risk factors, mode of symptom presentation, infarct pattern, procedure-related factors, and clinical outcomes, by univariate and multivariable logistic regression analysis.

RESULTS: Interobserver concordance showed good agreement for differentiating apical and body lesions ( $\kappa=0.745)$. Univariate analysis revealed that apical lesions $(n=108,54 \%)$ were associated with pseudo-occlusion ( $P=.027)$, older age $(P=.073)$, and alcohol intake $(P=.080)$, whereas body lesions ( $n=92,46 \%$ ) were associated with hyperlipidemia $(P=.001)$, a wedge-shaped cortical infarct pattern $(P=.057)$, and hyperperfusion syndrome $(P=.083)$. Multivariable logistic regression analysis adjusted by age revealed that hyperlipidemia $(P=.002 ; O R, 3.462 ; 95 \% \mathrm{Cl}, 1.595-7.515)$ and hyperperfusion ( $P=.026$; OR, 6.727; 95\% Cl, 1.261-35.894) were independent predictors of bodytype lesions.

CoNCLUSIONS: Atherosclerotic carotid bulb stenosis was found to have 2 distinct locations, body and apical. Hyperlipidemia and cortical wedge-shaped infarcts were more frequently associated with body than with apical stenosis at the time of presentation.

ABBREVIATIONS: $\mathrm{CCA}=$ common carotid artery; $\mathrm{Cl}=$ confidence interval; $\mathrm{ECA}=$ external carotid artery; FEV = forced expiratory volume; ICA = internal carotid artery; $\mathrm{mRS}=$ modified Rankin scale; OR = odds ratio; NASCET = North American Symptomatic Carotid Endarterectomy Trial; NIHSS $=$ National Institutes of Health Stroke Scale; WSS = wall shear stress

T he carotid bulb, the focally enlarged vascular structure just distal to the bifurcation of the CCA, is the major site of involvement in atherosclerotic stenosis. ${ }^{1}$ Vessel segments with low WSS and resultant flow stagnation at the carotid bulb appear to be at highest risk for development of atherosclerosis. ${ }^{2}$ Together with the condition of low WSS, a large lipid pool contributes to development of vulnerable plaque. ${ }^{3}$

Although high shear stress was initially reported to cause atherosclerosis, ${ }^{4}$ the involvement of low WSS associated with

Received August 11, 2009; accepted after revision November 20.

From the Department of Radiology and Research Institute of Radiology (S.-T.P., S.-O.P., S.J.K., D.C.S.), Department of Cardiology (S.W.P.), and Department of Neurology (J.S.K.), University of Ulsan, College of Medicine, Asan Medical Center, Songpa-Gu, Seoul, Korea; Department of Radiology (S.-T.P.), Soonchunhyang University Hospital, Yongsan-gu, Seoul, Korea; Department of Radiology (J.K.K.), Chung-Ang University College of Medicine, Seoul, Korea; and Department of Mechanical Engreering (K.H.Y.), Dankook University, Suji-Gu, Yongin-Si, Gyeonggi-Do, Korea.

This study was supported by a grant of the Korea Healthcare Technology R\&D Project, Ministry of Health and Welfare, Republic of Korea (A080201).

Please address correspondence to Dae Chul Suh, MD, Department of Radiology and Research Institute of Radiology, University of Ulsan, College of Medicine, Asan Medical Center, 388-1 Pungnap2-dong, Songpa-gu, Seoul 138-736, Korea; e-mail: dcsuh@ amc.seoul.kr

Indicates open access to non-subscribers at www.ajnr.org

DOI 10.3174/ajnr.A2000 hemodynamic parameters such as the WSS temporal gradient and cyclic strain high in the carotid bulb have more recently been associated with the formation of atherosclerosis. ${ }^{5}$ Men are more likely than women to develop maximum stenosis distal to the carotid bulb because of differences in absolute vessel size and the relative area of the ICA outflow compared with the CCA. ${ }^{6}$

We experienced 2 different patterns of plaque development in the carotid bulb in Korean patients. However, few reports on incidence, clinical features, or atherosclerotic risk factors have appeared. We therefore hypothesized that there are 2 different lesion locations in the carotid bulb, which may have distinct cardiovascular risk factors, presenting patterns, and clinical courses. Based on the morphologic definition of 2 locations of carotid bulb stenosis, we assessed their association with 13 atherosclerotic vascular risk factors, mode of symptom presentation, infarct patterns, procedure-related factors, and clinical outcomes.

\section{Materials and Methods}

We performed a retrospective analysis of 200 consecutive patients (men-to-women ratio, 165:35; mean age, 68 years; range, 42-87 years) who underwent carotid stent placement between March 2002 and September 2008 and from whom data were collected prospectively. Patients were included if they had symptomatic carotid stenosis 


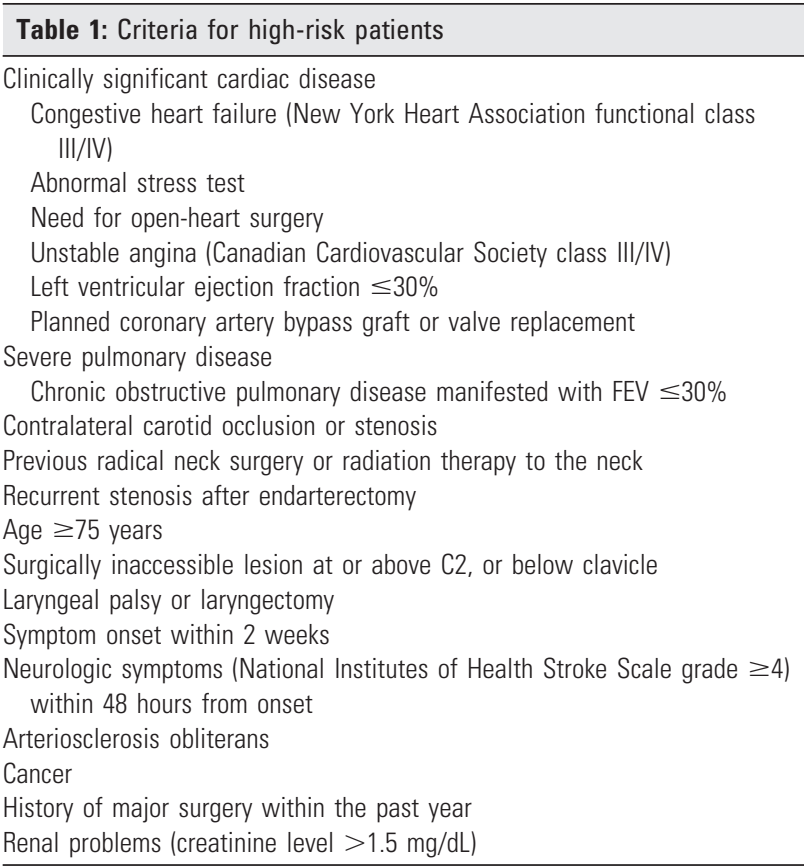

$\geq 50 \%$ according to NASCET criteria, whereby the diameter at the most stenotic point is compared with the reference diameter of the distal ICA beyond the narrowing and the poststenotic dilation. ${ }^{7}$ All patients were at high medical and surgical risk for carotid endarterectomy. ${ }^{8-10}$ The major eligibility criteria for high-risk patients are listed in Table 1. All patients were required to have at least one coexisting condition that potentially increased the risk posed by carotid endarterectomy.

We excluded patients with 1) nonatherosclerotic arterial diseases such as Takayasu arteritis and dissection, 2) acute symptom onset within 6 hours, and 3) stent placement following intra-arterial thrombolysis and/or angioplasty. This study was approved by our institutional review board.

\section{Angiographic Analysis of the Carotid Bulb}

The anatomic definitions are illustrated in Fig 1, with atheromatous plaque involvement of the carotid bulb categorized as an apical or body lesion (Figs 2 and 3)., 5, 11 Apical lesions were those in which the transitional zone of the bulb and the proximal cervical ICA segment (ie, the apical segment) were affected by plaque without or with minimal involvement of the body segment (Fig 2). Body lesions were those in which the transitional zone of the CCA and the bulb were affected by plaque located mainly in the body segment, thus causing the most severe stenosis at the body (Fig 3). We regarded involvement of the transitional zone between the CCA and the bulb, the most commonly involved site of atherosclerosis in the carotid bulb, as the most important differential point. ${ }^{12}$ Additionally, the main involvement area of plaque and the most severe stenosis were differentiated.

As plaque enlargement is regarded as an extended form of plaque, we defined an extended apical lesion as one in which apical plaque involved the body of the carotid bulb (Fig 4) and an extended body type of plaque as one in which the body plaque involved the apex (Fig 5). The main component with plaque involvement and the level of the most severe stenosis were considered as part of the extent of plaque for differentiation of the extended types. Each was distinguished by assessing at least 2 projections of the common carotid arterial injections. Two experienced neuroradiologists (S.J.K. and S.-T.P.), who

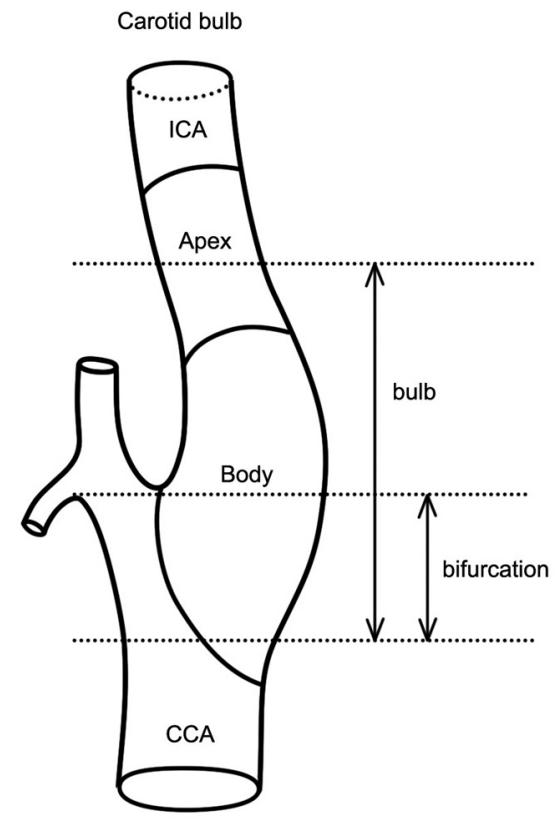

Fig 1. Anatomic definition of the carotid bulb.

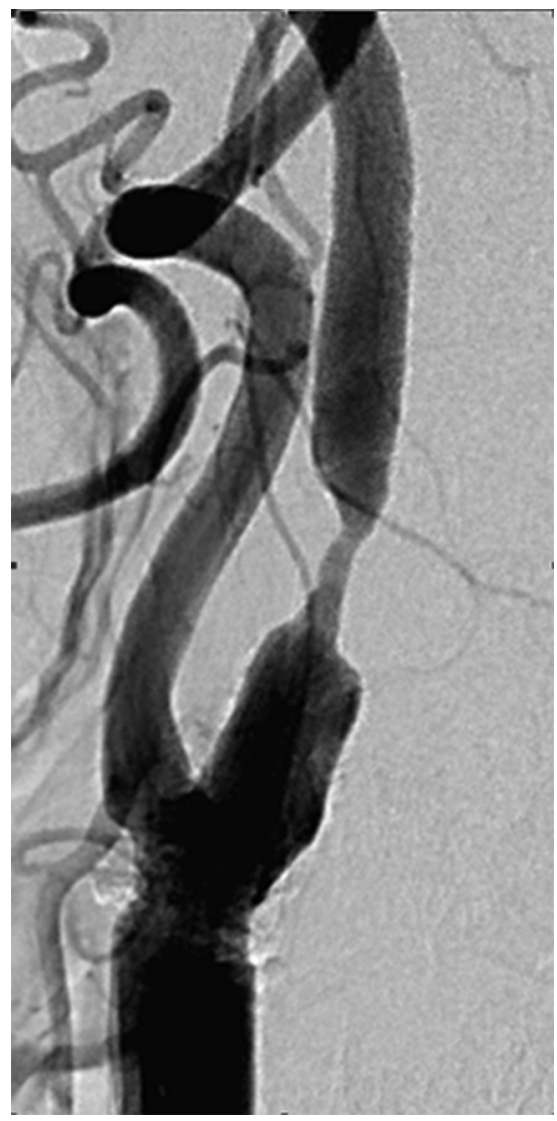

Fig 2. A 76-year-old man with an apical carotid bulb lesion detected before coronary artery bypass surgery. Brain MR imaging was normal (not shown). There was an apical form of severe stenosis in the carotid bulb. There was no carotid sinus reaction during the procedure.

were not involved in stent placement procedures and who were blinded to names, clinical histories, brain imaging data, and examination dates, independently reviewed all images, and interobserver agreement in lesion classifications was determined. 


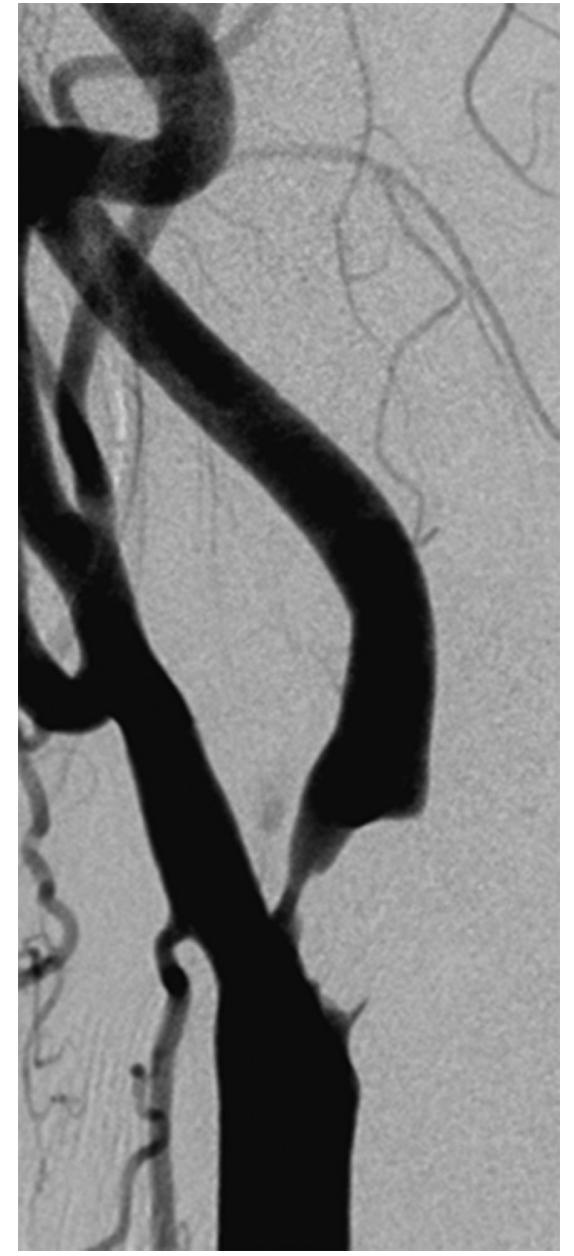

Fig 3. A 72-year-old woman with the body type of carotid bulb lesion presenting with right arm weakness and dysarthria occurring 20 days earlier. Body-type lesion in the right carotid bulb. There was a marked carotid sinus reaction during carotid stent placement.

As the mean carotid bulb lesion length in our patients was $20 \mathrm{~mm}$, we used this as the lesion length cutoff point. The degree of stenosis was classified as $50 \%-69 \%, 70 \%-79 \%, 80 \%-89 \%, 90 \%-94 \%$, or as a pseudo-occlusion $(>95 \%)$. Collateral circulation on angiography was evaluated by using Thrombolysis in Cerebral Infarction grading. ${ }^{13}$

\section{Analysis of Cardiovascular Risk Factors}

All variables were dichotomized for statistical analysis, including age ( $>67$ years vs $\leq 67$ years), sex (male vs female), hypertension (blood pressure $>140 / 90 \mathrm{mmHg}$ ), diabetes mellitus (fasting blood sugar level $>126 \mathrm{mg} / \mathrm{dL}$, postprandial 2-hour blood sugar level $>200 \mathrm{mg} /$ $\mathrm{dL}$ ), hyperlipidemia (total cholesterol $\geq 220 \mathrm{mg} / \mathrm{dL}$ or $\mathrm{LDL} \geq 130$ $\mathrm{mg} / \mathrm{dL}$ ), smoking (currently smoking or ex-smoker for $<6$ months), homocysteine level ( $\geq 15 \mu \mathrm{mol} / \mathrm{L}),{ }^{14}$ high-sensitivity C-reactive protein concentration $(\geq 0.6 \mathrm{mg} / \mathrm{dL}),{ }^{15}$ and body mass index $(\geq 25$ $\left.\mathrm{kg} / \mathrm{m}^{2}\right){ }^{16}$

\section{Analysis of Symptom Presentation and MR Imaging Infarct Patterns}

The severity of initial symptoms was evaluated by using the NIHSS. ${ }^{17}$ The presenting symptom pattern of each patient was classified as a transient ischemic attack, stroke, or no symptoms. The symptom on- set period before the stent placement procedure was classified as within 1 day, 1 week, 1 month, or $>1$ month.

All patients underwent brain MR imaging examination before carotid stent placement. Cerebral ischemic changes or infarctions on MR imaging at the time of symptom presentation were classified according to the lesion distribution of high-signal intensities on diffusion- or T2-weighted images. ${ }^{18,19}$ The topography of each ischemic lesion was determined by using published templates and classified as a cortical wedge or territorial infarct, a perforator infarct, or a borderzone infarct. ${ }^{18}$ All parenchymal lesions were classified according to their longest dimension as large $(>20 \mathrm{~mm})$, medium $(10-20 \mathrm{~mm})$, and small $(<10 \mathrm{~mm})$. Lesion number was classified as 1 or multiple.

\section{Procedure-Related Factors and Clinical Outcomes}

Procedure-related factors included the type of stent (ie, open vs closed) and use of a protection device. Hemodynamic disturbances during and after stent placement included periprocedural hypotension (systolic blood pressure $<90 \mathrm{~mm} \mathrm{Hg}$ ), bradycardia (heart rate $<50$ beats/s), heart rate fluctuation ( $>20$ beats/s), and hypertension (>160 mm Hg). Hyperperfusion was diagnosed when MR imaging showed increased perfusion in the brain vascular territory, corresponding to the development of symptoms within 1 month after stent placement, or a hemorrhage in the ipsilateral vascular territory not otherwise explained.

Patients were followed up by Doppler ultrasonography or cerebral angiography 6-12 months after the procedure. Restenosis ( $>50 \%$ ) was defined as a reduction in the luminal diameter by using NASCET criteria on cerebral angiography, or by using B-mode sonography and/or peak systolic velocity $(>300 \mathrm{~cm} / \mathrm{s}$ or $>4$-fold the ICA/CCA ratio) on Doppler sonography. ${ }^{20}$ Events after stent placement included minor stroke, major stroke, or death. Minor stroke was defined as a new, nondisabling neurologic deficit or a 3-point increase in the NIHSS, either of which completely resolved within 30 days. ${ }^{21}$ Major stroke was defined as a new neurologic deficit with a 4-point increase in NIHSS persisting for $>30$ days. The final outcome at 6 months was determined by using the mRS, with good outcome scoring $\leq 2$ and poor outcomes $\geq 3 .{ }^{22}$

\section{Statistical Analysis}

Interobserver agreement on angiographic lesion location (ie, apical vs body) was assessed by using Cohen $\kappa$ statistics. Lesion location was regarded as a dependent variable. We extracted explanatory variables from risk factors and angiographic results. Univariate analysis, by using the $\chi^{2}$ or Fischer exact test, was performed to determine the association between lesion location and the explanatory variables. Continuous variables were converted to nominal variables according to median or mean values. Explanatory variables showing $P$ values $<.1$ on univariate analysis were selected for multivariable logistic regression analysis, which evaluated interaction among the explanatory variables. All $P$ values were calculated by using the 2 -tailed test. A $P$ value of $<.05$ was considered significant. Calculations were performed by using SPSS 14.0 for Windows (SPSS, Chicago, Illinois)

\section{Results}

\section{Angiographic Analysis of the Carotid Bulb}

Interobserver concordance showed good agreement for differentiating lesions in the apex and body of the carotid bulb $(\kappa=$ 0.745). Of the 200 patients, 108 had apical and 92 had body lesions. Pseudo-occlusion showed significant association with 

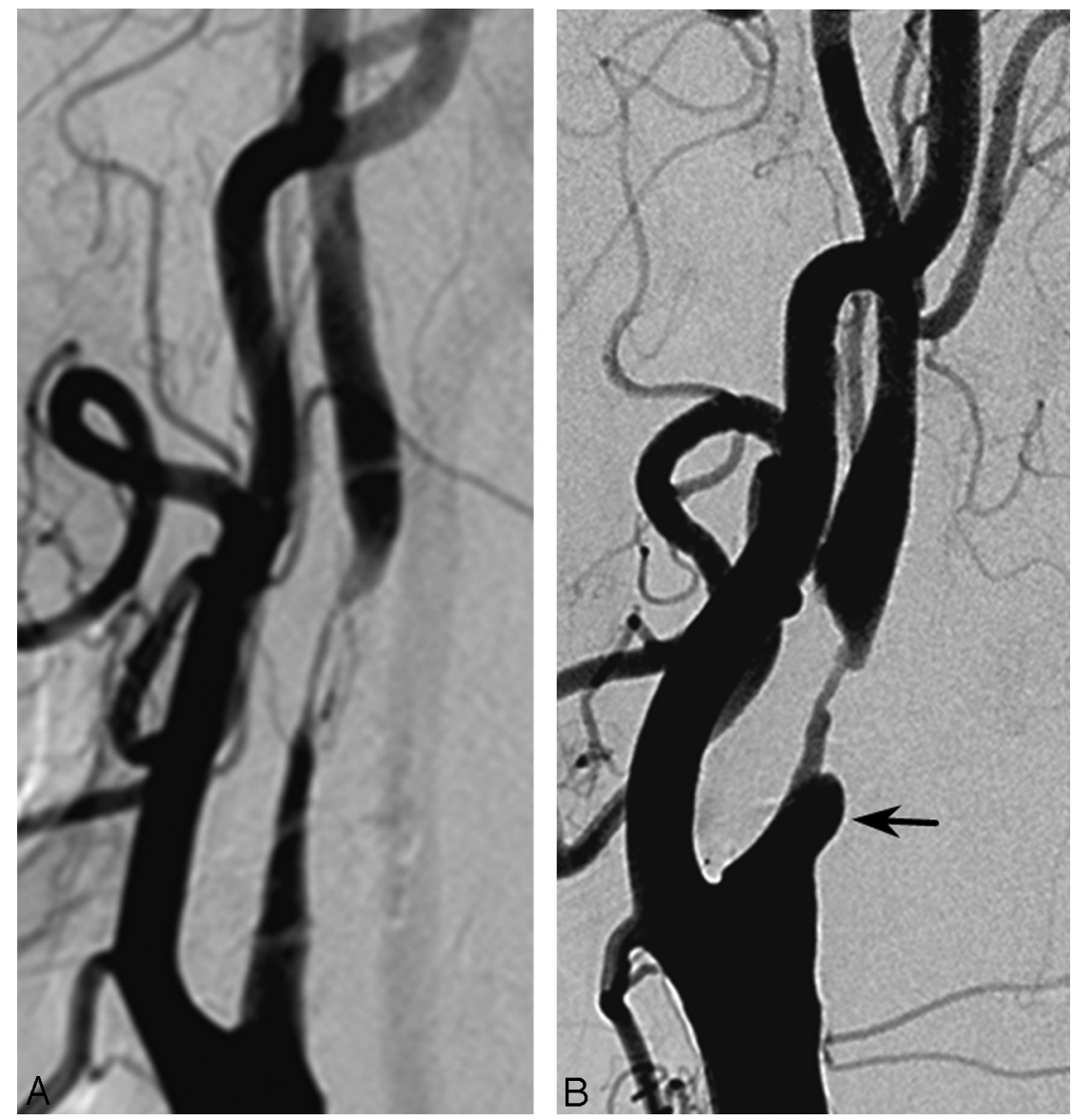

Fig 4. Extended apical type stenosis. A diffuse plaque extends to the bulb area $(A)$ and/or reveals ulceration (arrow) $(B)$.

the apical type $(P=.027)$ on univariate analysis. The length of stenosis and collateral circulation was not associated with either type. Bilateral lesions were identified in 29 patients (15\%). The concordance rate of the same type of lesion in patients with bilateral stenosis was $90 \%$. Of the 50 extended lesions in 45 patients $(23 \%)$, 33 were apical and 17 were of the bulb type. When extended lesions were excluded, interobserver concordance also showed good agreement $(\kappa=0.798)$.

\section{Analysis of Cardiovascular Risk Factors}

Univariate analysis of 13 atherosclerotic cardiovascular risk factors showed a significant association between hyperlipidemia and body-type lesions $(P=.001$, Table 2$)$. Age $(P=.073)$ and alcohol consumption $(P=.080)$ were marginally related to apical-type lesion occurrence.

\section{Analysis of Symptom Presentation and MR Imaging Infarct Patterns}

Presenting symptom patterns, including severity, presentation, and symptom onset to procedure, were not associated with either type of lesion. Assessment of brain parenchymal infarct pattern at the time of symptom presentation showed a significant association between cortical-wedge infarcts and body-type lesions $(P=.057$, Fig 3 and Table 3$)$.

\section{Procedure-Related Factors and Clinical Outcomes}

Closed-cell-type stents and protection devices were more frequently used for body than for apical lesions. These proce- dure-related factors were at the discretion of individual operators. Hyperperfusion syndrome $(P=.083)$ was marginally related to body lesion occurrence, whereas frequent carotid sinus reactions were not $(P=.228$, Table 4$)$.

Six-month follow-up mRS, restenosis, and events, as well as $\mathrm{mRS}$ after the procedure, were not associated with either lesion type (Table 4 ).

\section{Multivariable Logistic Regression Analysis}

Explanatory variables showing $P$ values of $<.1$ on univariate analysis included hyperlipidemia $(P=.001)$, pseudoocclusion $(P=.027)$, alcohol use $(P=.080)$, age $(P=.073)$, and hyperperfusion syndrome $(P=.083)$. Multivariable logistic regression analysis adjusted by age showed that hyperlipidemia $(P=$ .002; OR, 3.462; 95\% CI, 1.595-7.515) and hyperperfusion $(P=.026$; OR, 6.727; 95\% CI, 1.261-35.894) were independent predictors of body-type lesions.

\section{Discussion}

The subtyping of atherosclerotic carotid stenosis is supported by some clinical factors. Whereas body lesions are more frequent than are apical lesions in patients with hyperlipidemia and postprocedural hyperperfusion syndrome, apical lesions are more frequent in older patients and those with pseudoocclusions. The association between hyperlipidemia and body lesion corresponds to the classic pathogenesis of atherosclerotic plaque in the carotid bulb, especially in the body portion of the hemodynamically low WSS area. ${ }^{3}$ 

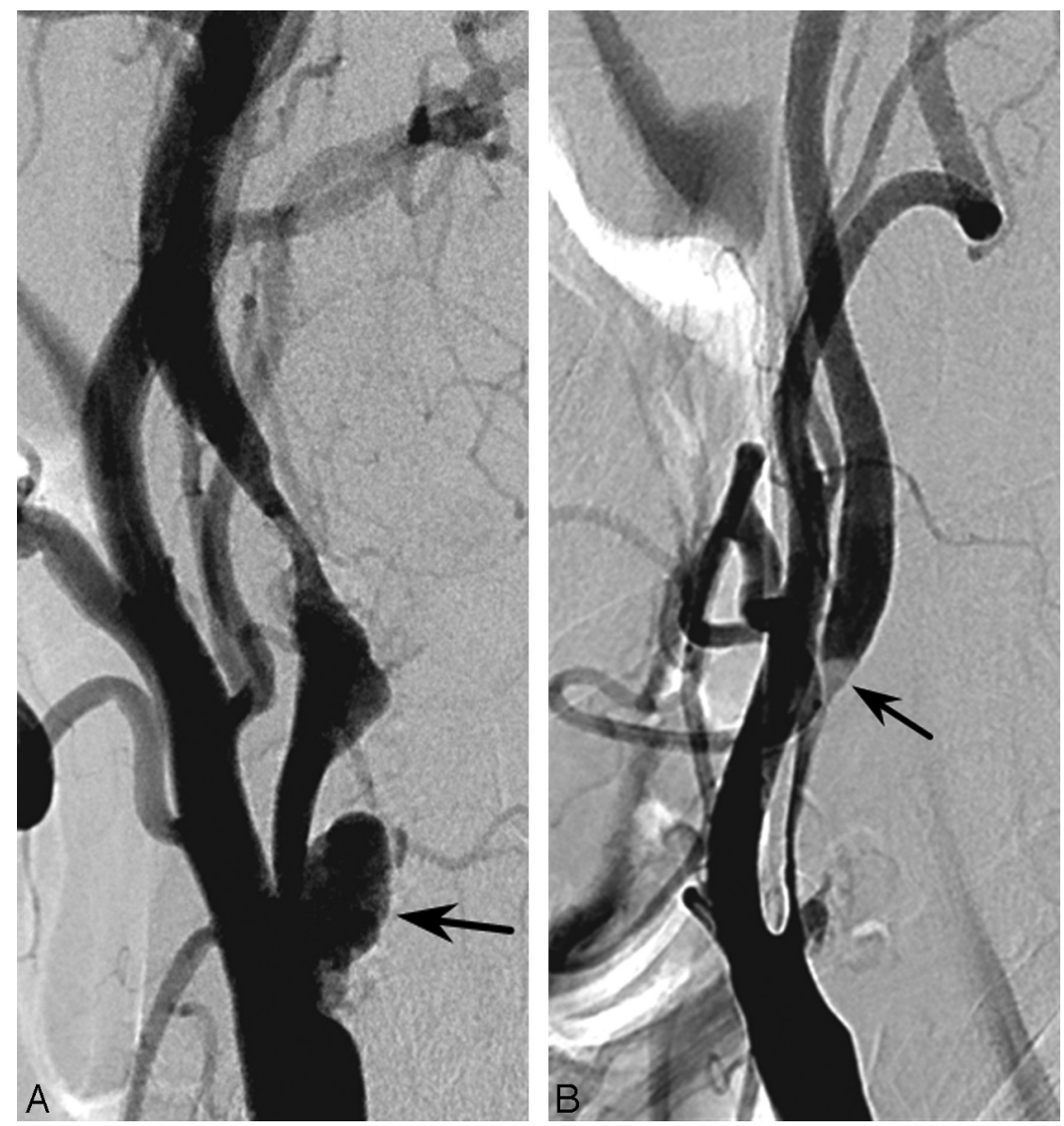

Fig 5. Extended bulb type stenosis. A primary bulb lesion extends to the apex with ulcerative bulb plaque $(A)$ and ulceration associated with a luminal filling defect (arrow) (B).

\begin{tabular}{lccc}
\hline Table 2: Demographic features and cardiovascular risk factors \\
\hline & $\begin{array}{c}\text { Apical Lesion } \\
(n=108)\end{array}$ & $\begin{array}{c}\text { Body Lesion } \\
(n=92)\end{array}$ & $\begin{array}{c}P \\
\text { Value }\end{array}$ \\
\hline Age (>67 years) & 63 & 42 & .073 \\
Gender (male) & 91 & 74 & .478 \\
Hypertension (presence) & 80 & 72 & .490 \\
Diabetes mellitus (presence) & 43 & 35 & .798 \\
Cardiac disease (presence) & 33 & 21 & .220 \\
Hyperlipidemia (presence) & 12 & 28 & .001 \\
Family history (presence) & 20 & 17 & .994 \\
Previous stroke history (presence) & 32 & 32 & .436 \\
Smoking (yes) & 38 & 35 & .676 \\
Alcohol use (yes) & 58 & 38 & .080 \\
Homocysteine ( $\geq 15 \mu$ mol/L) & 34 & 32 & .948 \\
High-sensitivity C-reactive protein & 31 & 26 & .857 \\
$\quad$ ( $\geq 0.6$ mg/dL) & & & \\
Body mass index ( $\geq 25)$ & 34 & 30 & .865 \\
\hline
\end{tabular}

\begin{tabular}{lccc}
\hline Table 3: Factors related to brain infarcts & & \\
\hline & $\begin{array}{c}\text { Apical Lesion } \\
(n=56)\end{array}$ & $\begin{array}{c}\text { Body Lesion } \\
(n=55)\end{array}$ & $\begin{array}{c}P \\
\text { Value }\end{array}$ \\
\hline $\begin{array}{l}\text { Infarct pattern } \\
\quad \text { Cortical wedge/territorial }\end{array}$ & 13 & 22 & .057 \\
$\quad$ lesion & & & \\
$\quad$ Others & 43 & 33 & .245 \\
Infarct size & & & \\
$\quad$ Large $(\geq 20 \mathrm{~mm})$ & 15 & 21 & \\
$\quad$ Medium $(10-20 \mathrm{~mm})$ & 13 & 7 & .167 \\
$\quad$ Small $(<10 \mathrm{~mm})$ & 28 & 27 & \\
Number of infarcts & & & \\
$\quad$ Single & 14 & 8 & \\
$\quad$ Multiple & 42 & 47 & \\
\hline
\end{tabular}

Table 4: Factors related to stenting procedure and clinical outcomes after procedure

The posterior wall of the carotid bulb (ie, the focally enlarged vascular structure just distal to the bifurcation of the CCA) is the primary site of involvement of atherosclerotic stenosis. Embryologically, the cervical ICA at the common carotid bifurcation, the bulb, and the CCA originate from the third aortic arch. ${ }^{23,24}$ Despite the body being the most common site of atherosclerotic involvement, we found that carotid stenosis affected the apex to an extent similar to that of the body. This may be attributable to ethnic factors. Our study included only Korean patients; thus, the ratio of apical-tobody stenosis may differ in other populations, including West-

\begin{tabular}{lccr}
\hline & $\begin{array}{c}\text { Apical Lesion } \\
(n=108)\end{array}$ & $\begin{array}{c}\text { Body Lesion } \\
(n=92)\end{array}$ & $\begin{array}{c}P \\
\text { Value }\end{array}$ \\
\hline Carotid sinus reaction & 68 & 65 & .228 \\
Hyperperfusion syndrome & 2 & 7 & .083 \\
Restenosis at 6 months & 6 & 5 & 1.000 \\
Events within 6 months & 12 & 9 & .746 \\
\hline
\end{tabular}

ern patients. There may be a greater tendency toward apicaltype lesions in Koreans, as bulb-type lesions are more common in Western populations and carotid stenosis is less common than is intracranial stenosis in Koreans. ${ }^{25}$ There may 


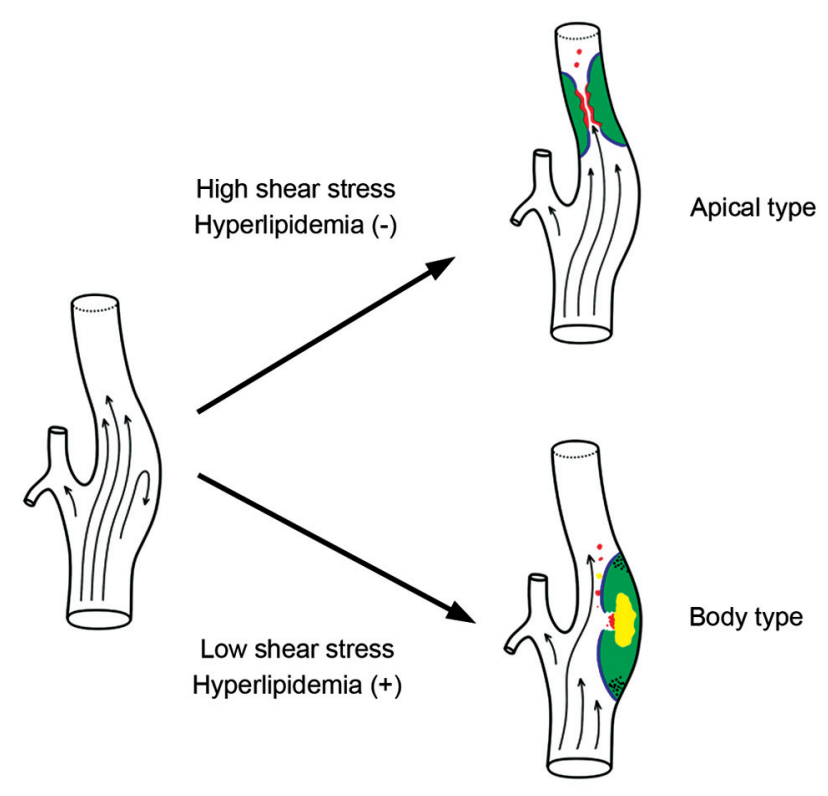

Fig 6. Concept diagram of carotid bulb lesion type; the apical and body lesions.

also be racial/ethnic differences in the anatomy of the carotid bifurcation, in that African Americans were found to have a proportionally smaller ICA and a larger ECA than did whites or Caribbean Hispanics. ${ }^{26}$ Thus, hemodynamic factors, ${ }^{5}$ as well as sex differences in carotid bifurcation anatomy and the distribution of atherosclerotic plaque, ${ }^{6}$ suggest that the 2 distinct locations of carotid bulb stenosis may correspond to 2 different flow mechanisms, low WSS in the body type and high WSS in the apical type.

Although previous hemodynamic studies assumed that low WSS caused atherosclerosis in the carotid bulb, the role of high WSS in atherosclerosis is unclear, and high WSS may or may not be a causative factor (Fig 6). ${ }^{2,4}$ Low WSS is thought to induce plaques, whereas high WSS is considered to be involved in the shear stress that causes plaque rupture. ${ }^{2,27,28}$ The presence of an atherosclerotic lesion in the body of the carotid bulb can be explained by classical hemodynamic theory, that is, a low WSS in conjunction with hyperlipidemia. ${ }^{3}$ Alternatively, the apex of the carotid bulb has been found to have 2 other characteristic hemodynamic factors, a high WSS temporal gradient and an elevated degree of cyclic strain, both of which are involved in atherosclerosis. ${ }^{11,29,30}$ Additional clinical and hemodynamic studies may be needed to determine the exact causes and possible mechanisms underlying the differences seen.

Our results suggest that stenosis location may affect the frequency of the carotid sinus reaction triggered by stimulation of the carotid baroreceptor in the carotid body, reducing the sympathetic tone and increasing vagal activity, resulting in a decrease in blood pressure and heart rate. ${ }^{31,32}$ The distance between the carotid bifurcation and the maximum stenotic lesion $(\leq 10 \mathrm{~mm})$ was reported to be an independent risk factor for carotid artery stent placement in 31 patients, as lesions with maximum stenosis $>10 \mathrm{~mm}$ from the carotid bifurcation were associated with a low incidence of hypotension. ${ }^{33}$ Hyperperfusion syndrome ${ }^{34}$ occurred frequently in our patients with body-type lesions, suggesting that the carotid body has a hemodynamic effect on the ipsilateral cerebral circulation.
Several attempts have been made to document anatomic differences attributable to age and sex. The ICA, relative to both the ECA and the CCA, is larger in women than in men, suggesting that men are more likely to show maximum stenosis distal to the carotid bulb, thus at the apex, whereas women are more likely to present with stenosis of the ECA. ${ }^{6}$ However, the cited study excluded patients with severe $(>50 \%)$ stenosis on NASCET (identical to $>70 \%$ on European Carotid Surgery Trial), because of the resulting secondary deformity. ${ }^{6,35} \mathrm{We}$ observed no difference by sex in the site of stenosis, suggesting that the earlier distinction may have been unique to the cohort evaluated.

\section{Conclusions}

We found that atherosclerotic carotid bulb stenosis tended to occur in 2 distinct locations, body and apical. The latter lesions were associated with pseudo-occlusion, older age, and alcohol intake, whereas body lesions were accompanied by hyperlipidemia, a wedge-shaped cortical infarct pattern, and hyperperfusion syndrome.

\section{Acknowledgments}

We acknowledge the assistance of Sun Moon Whang and Eun Hye Kim in patient data collection, and Yun Gyeong Jeong for manuscript preparation. We thank Bonnie Hami for her English editorial assistance.

\section{References}

1. Pujia A, Rubba P, Spencer MP. Prevalence of extracranial carotid artery disease detectable by echo-Doppler in an elderly population. Stroke 1992;23: $818-22$

2. Shaaban AM, Duerinckx AJ. Wall shear stress and early atherosclerosis: a review. AJR Am J Roentgenol 2000;174:1657-65

3. Cheng C, Tempel D, van Haperen R, et al. Shear stress-induced changes in atherosclerotic plaque composition are modulated by chemokines. J Clin Invest 2007;117:616-26

4. Fry DL. Certain histological and chemical responses of the vascular interface to acutely induced mechanical stress in the aorta of the dog. Circ Res 1969;24:93-108

5. Younis HF, Kaazempur-Mofrad MR, Chan RC, et al. Hemodynamics and wall mechanics in human carotid bifurcation and its consequences for atherogenesis: investigation of inter-individual variation. Biomech Model Mechanobiol 2004;3:17-32

6. Schulz UG, Rothwell PM. Sex differences in carotid bifurcation anatomy and the distribution of atherosclerotic plaque. Stroke 2001;32:1525-31

7. North American Symptomatic Carotid Endarterectomy Trial Collaborators. Beneficial effect of carotid endarterectomy in symptomatic patients with high-grade carotid stenosis. N Engl J Med 1991;325:445-53

8. White CJ, Iyer SS, Hopkins LN, et al. Carotid stenting with distal protection in high surgical risk patients: the BEACH trial 30 day results. Catheter Cardiovasc Interv 2006;67:503-12

9. Yadav JS, Wholey MH, Kuntz RE, et al. Protected carotid-artery stenting versus endarterectomy in high-risk patients. N Engl J Med 2004;351:1493-501

10. Suh DC, Kim JK, Choi JW, et al. Intracranial stenting of severe symptomatic intracranial stenosis: results of 100 consecutive patients. AJNR Am J Neuroradiol 2008;29:781-85

11. Salzar RS, Thubrikar MJ, Eppink RT. Pressure-induced mechanical stress in the carotid artery bifurcation: a possible correlation to atherosclerosis. J Biomech 1995;28:1333-40

12. Sundberg J Localisation of atheromatosis and calcification in the carotid bifurcation: a post mortem radiographic investigation. Acta Radiol Diagn (Stockh) 1981;22:521-28

13. Higashida RT, Furlan AJ. Trial design and reporting standards for intra-arterial cerebral thrombolysis for acute ischemic stroke. Stroke 2003;34:e109-37

14. Coull BM, Malinow MR, Beamer N, et al. Elevated plasma homocyst(e)ine concentration as a possible independent risk factor for stroke. Stroke 1990;21: 572-76

15. Yeh ETH, Willerson JT. Coming of age of C-reactive protein: using inflammation markers in cardiology. Circulation 2003;107:370-71 
16. Balkau B, Valensi P, Eschwege E, et al.. A review of the metabolic syndrome. Diabetes Metab 2007;33:405-13

17. del Zoppo GJ, Higashida RT, Furlan AJ, et al. PROACT: a phase II randomized trial of recombinant pro-urokinase by direct arterial delivery in acute middle cerebral artery stroke. Stroke 1998;29:4-11

18. Lee DK, Kim JS, Kwon SU, et al. Lesion patterns and stroke mechanism in atherosclerotic middle cerebral artery disease: early diffusion-weighted imaging study. Stroke 2005;36:2583-88

19. Lee $\mathrm{PH}, \mathrm{Oh} \mathrm{SH}, \mathrm{Bang} \mathrm{OY}$, et al. Isolated middle cerebral artery disease: clinical and neuroradiological features depending on the pathogenesis. J Neurol Neurosurg Psychiatry 2004;75:727-32

20. Setacci C, Chisci E, Setacci F, et al. Grading carotid intrastent restenosis: a 6-year follow-up study. Stroke 2008;39:1189-96

21. Goldstein LB, Samsa GP. Reliability of the National Institutes of Health Stroke Scale: extension to non-neurologists in the context of a clinical trial. Stroke 1997;28:307-10

22. Sulter G, Steen C, Jacques De K. Use of the Barthel index and modified Rankin scale in acute stroke trials. Stroke 1999;30:1538-41

23. Lasjaunias $P$, Berenstein A, Brugge K. Skull base and maxillofacial region. In: Surgical Neuro-angiography, 2nd ed. New York: Springer-Verlag; 2001:261-386

24. Kameda Y. Hoxa3 and signaling molecules involved in aortic arch patterning and remodeling. Cell Tissue Res 2009;336:165-78

25. Suh DC, Lee SH, Kim KR, et al. Pattern of atherosclerotic carotid stenosis in Korean patients with stroke: different involvement of intracranial versus extracranial vessels. AJNR Am J Neuroradiol 2003;24:239-44

26. Koch S, Nelson D, Rundek $\mathrm{T}$, et al. Race-ethnic variation in carotid bifurcation geometry. J Stroke Cerebrovasc Dis 2009;18:349-53
27. Helderman F, Segers D, de Crom R, et al. Effect of shear stress on vascular inflammation and plaque development. Curr Opin Lipidol 2007;18:527-33

28. Groen HC, Gijsen FJ, van der Lugt A, et al. Plaque rupture in the carotid artery is localized at the high shear stress region: a case report. Stroke 2007;38: $2379-81$

29. Masawa N, Glagov S, Zarins CK. Quantitative morphologic study of intimal thickening at the human carotid bifurcation, I: Axial and circumferential distribution of maximum intimal thickening in asymptomatic, uncomplicated plaques. Atherosclerosis 1994;107:137-46

30. Friedman MH, Hutchins GM, Bargeron CB, et al. Correlation of human arterial morphology with hemodynamic measurements in arterial casts. JBiomech Eng 1981;103:204-07

31. Doux JD, Yun AJ. The link between carotid artery disease and ischemic stroke may be partially attributable to autonomic dysfunction and failure of cerebrovascular autoregulation triggered by Darwinian maladaptation of the carotid baroreceptors and chemoreceptors. Med Hypotheses 2006;66:176-81

32. Qureshi AI, Luft AR, Sharma M, et al. Frequency and determinants of postprocedural hemodynamic instability after carotid angioplasty and stenting. Stroke 1999;30:2086-93

33. Nonaka T, Oka S, Miyata K, et al. Prediction of prolonged postprocedura hypotension after carotid artery stenting. Neurosurgery 2005;57:472-77

34. Abou-Chebl A, Yadav JS, Reginelli JP, et al. Intracranial hemorrhage and hyperperfusion syndrome following carotid artery stenting: risk factors, prevention, and treatment. J Am Coll Cardiol 2004;43:1596-601

35. Rothwell PM, Gibson RJ, Slattery J, et al. Equivalence of measurements of carotid stenosis: a comparison of three methods on 1001 angiograms. European Carotid Surgery Trialists' Collaborative Group. Stroke 1994;25:2435-9 